Check for updates

Cite this: J. Mater. Chem. C, 2019, 7, 12312

Received 19th July 2019,

Accepted 6th September 2019

DOI: $10.1039 / c 9 t c 03936 h$

rsc.li/materials-c

\title{
An emerging Janus MoSeTe material for potential applications in optoelectronic devices $\dagger$
}

\author{
Xiaoyong Yang, (D) ab Deobrat Singh, (D) *b Zhitong $\mathrm{Xu}^{\mathrm{a}}{ }^{\mathrm{a}}$ Ziwei Wang ${ }^{\mathrm{a}}$ and \\ Rajeev Ahuja (D) *bc
}

\begin{abstract}
Motivated by the extraordinary physical and chemical properties of Janus transition-metal dichalcogenides (TMDs) due to the change of the crystal field originating from their asymmetry structures, the electronic and optical properties of the MoSeTe monolayer in $2 \mathrm{H}$ and $1 \mathrm{~T}$ phases are systematically studied by firstprinciples calculations, and a detailed comparison with the parental $\mathrm{MoSe}_{2}$ and $\mathrm{MoTe}_{2}$ monolayer is made. It is found that $2 \mathrm{H}$-MoSeTe exhibits a direct bandgap of $1.859 \mathrm{eV}$ and an indirect band gap of $0.391 \mathrm{eV}$ in the $1 T$ phase, resulting in a different way to interact with sunlight. Besides, the obtained results show that the SOC has little effects on the band gaps. The calculated optical properties show a significant red shift from the $\mathrm{MoSe}_{2}$ to MoSeTe to MoTe 2 monolayer. However, a blue shift is observed from the in-plane to out-ofplane direction. Moreover, both electron-electron and electron-hole correlation effects are considered for obtaining the optical spectra of systems by $G_{0} W_{0}$ and $G_{0} W_{0}+$ BSE approaches. Besides, the absorption coefficient value reaches up to $1 \times 10^{6} \mathrm{~cm}^{-1}$ in both phases, implying the high efficiency in the utilization of solar energy for the MoSeTe monolayer. Additionally, the 1T-MoSeTe monolayer is a good hot mirror material in that its maximum reflectivity could reach up to $51 \%$ in the infrared region. Additionally, the average optical absorbance of the Janus MoSeTe monolayer in the visible light region is calculated to be about $2 \%$ and the corresponding average transmittance is around $80 \%$. More importantly, the difference in the optical response for the two side surfaces is considered in our work due to the intrinsic asymmetric structure of Janus MoSeTe. These results not only predict the great potential application of Janus MoSeTe in optoelectronics-electronic devices, but may enable the discovery of new optical science and the realization of various light emissions, detection, modulation and manipulation functions of specific frequencies.
\end{abstract}

\section{Introduction}

The ability of materials to absorb, reflect and act upon light illumination plays a fundamental and important role for scientists to develop optoelectronics-electronic devices. Following the discovery of graphene, ${ }^{1-3}$ 2D materials have attracted increasing attention due to a range of optical, electronic and physical properties originating from the bulk counterpart to monolayer transition. Taking graphene as an example, it has a low frequency absorbance defined by the universal analytical constant $(\pi / 137 \sim 0.023),{ }^{3}$ making it a promising

\footnotetext{
${ }^{a}$ National Collaborative Innovation Center for Nuclear Waste and Environmental Safety, Southwest University of Science and Technology, Mianyang 621010, China

${ }^{b}$ Condensed Matter Theory Group, Materials Theory Division, Department of Physics and Astronomy, Uppsala University, Box 516, 75120 Uppsala, Sweden. E-mail: deobratsingh9@gmail.com, rajeev.ahuja@physics.uu.se; Fax: +46 184713524; Tel: +46728772897

${ }^{c}$ Applied Materials Physics, Department of Materials and Engineering, Royal Institute of Technology (KTH), S-100 44 Stockholm, Sweden $\dagger$ Electronic supplementary information (ESI) available. See DOI: 10.1039/ c9tc03936h
}

candidate for various light detection, modulation, and manipulation functions. However, the metallic feature of graphene prevents its application in efficient light emitting devices. In contrast, transitionmetal dichalcogenides (TMDs), such as $\mathrm{MoS}_{2}$ and $\mathrm{WSe}_{2}$, evolving from indirect bandgap to direct bandgap transitions, could absorb up to $10 \%$ at excitonic resonances in the near-infrared wavelength range, arising from the reduced dielectric screening due to the absence of interlayer interactions in comparison with their layered counterparts. ${ }^{4,5}$ Hexagonal boron nitride (hBN), an important type of 2D material, possesses a large bandgap of about $6 \mathrm{eV}$, making it an excellent dielectric. ${ }^{6,7}$ Another distinguished property of $2 \mathrm{D}$ materials is that they could exhibit good compatibility with the present device design and fabrication standards in the electronic industry due to their high mechanical strength and flexibility. ${ }^{8,9}$ In all, many $2 \mathrm{D}$ materials interact with light strongly and exhibit an extraordinary optical response, such as absorbance and reflectance in different spectral regions (mid-, near-infrared (IR), visible, near-, mid-, deep-ultraviolet (UV)), which opens up prospects for 2D material exploration for use as absorbers, reflectors, and modulators in 
electronic, optoelectronic, thermoelectric, quantum, sensing, flexible devices, and in many other fields.

To date, there have been over 100 members of 2D materials discovered and explored, including TMDs, ${ }^{10,11}$ MXenes, ${ }^{12}$ silicone ${ }^{13}$ germanene,${ }^{14}$ stanene, ${ }^{15}$ phosphorene, ${ }^{8}$ borophene,${ }^{16}$ indiene, ${ }^{17}$ organic $2 \mathrm{D}$ polymers,${ }^{18} 2 \mathrm{D}$ perovskites, ${ }^{19} 2 \mathrm{D}$ oxides,${ }^{20} 2 \mathrm{D}$ hydroxides, ${ }^{21}$ etc. Among them, TMDs have great potential for application in optical nanodevices, such as photodiodes, solar cells, photocatalytic cells, phototransistors, and photodetectors, due to their inversion in-plane symmetry breaking and strong spin-orbit coupling, which lead to valley-selective circular dichroism. Very recently, a new type of TMDs, called the Janus MoSSe monolayer, has been successfully synthesized via two different synthetic pathways by fully replacing the top S layer with Se, resulting in an out-of-plane structural asymmetry. ${ }^{22,23}$ One method employed by Lu et al. is the use of a modified CVD grown $\mathrm{MoS}_{2}$ monolayer, where one layer of $\mathrm{S}$ atoms is replaced by $\mathrm{H}$ atoms and then followed by thermal selenization, and thus a structurally stable Janus MoSSe monolayer is formed. ${ }^{22}$ They have confirmed the existence of vertical dipoles from the second harmonic generation and piezoresponse force microscopy measurements. ${ }^{22}$ Another strategy to synthesize MoSSe starts from the CVD as-grown $\mathrm{MoSe}_{2}$ monolayers on a $\mathrm{SiO}_{2} / \mathrm{Si}$ substrate, followed by sulfurization on the top layer Se in a controlled substitutional reaction with vaporized sulfur. ${ }^{23}$ Based on $a b$ initio calculations, the large Zeeman- and Rashba-type spin splitting and strong in-plane and vertical piezoelectric effects are systemically studied for Janus $\operatorname{MXY}(\mathrm{M}=\mathrm{Mo}, \mathrm{W}$, and $\mathrm{X} / \mathrm{Y}=\mathrm{S}, \mathrm{Se}$, and Te$),{ }^{24-26}$ which makes a main contribution to light-electricity interconversion. Additionally, the structures and electronic properties of $2 \mathrm{D}$ TMD alloys with halfto-half mixing of S and Se for Sc, Ti, Cr, Mn, Fe, Zr, Mo and W were studied for the first time. ${ }^{27}$ However, currently, a comprehensive study to estimate the transmittance, absorbance, and reflectance (TAR) of 2D materials is very scarce, which is essential for analyzing their efficiency as ultrathin reflectors, transmitters or absorbers. For example, one may think that 2D materials are so thin that they should appear to be essentially transparent with negligible reflection and absorption. Surprisingly, Yakobson et al. found that each 2D material has an expressive optical signature with a large portion of light of a particular wavelength being absorbed or reflected. ${ }^{28}$ Correspondingly, are there any limitations on TAR in these 2D materials as absorbing/reflecting in bulk forms? Furthermore, we all know that dimensionality plays a fundamental and important role for physical and chemical properties of materials. Then being at the ultimate limit of atomic size in one direction, 2D materials can be used as coating materials in some more sophisticated structures such as metamaterials, where a nontrivial layerdependent optical response may emerge in which the nonlinear optical effects become non-negligible, and accounting for these will certainly require further investigation. This will stimulate many research studies on the optical properties for 2D materials in future.

Motivated by the above attractive properties and significant problems of 2D materials, the electronic structures and optical properties of the novel Janus MoSeTe monolayer in $2 \mathrm{H}$ and 1T phases are systematically studied in our work based on first-principles calculations, with an aim to identify and quantify the optical response in comparison with the parental $\mathrm{MoSe}_{2}$ and $\mathrm{MoTe}_{2}$ over a wide optical spectrum. It is found that the $2 \mathrm{H}-\mathrm{MoSeTe}$ monolayer exhibits a direct bandgap of $1.859 \mathrm{eV}$ and an indirect gap of $0.251 \mathrm{eV}$ in the $1 \mathrm{~T}$ structure, which may trigger plasmons absorbing light at different frequencies and emitting light at other frequencies. This is indeed the case. Our calculations verify that the 1T-MoSeTe monolayer is highly reflective of mid-infrared light with a maximum reflectivity of $51 \%$, enabling the 1T-MoSeTe monolayer to be a good hot mirror material used in optical fibers. However, the $2 \mathrm{H}$ phase could reflect more light from visible to ultraviolet. Besides, the absorption coefficients of both proposed configurations could reach up to $1 \times 10^{6} \mathrm{~cm}^{-1}$. We also evaluate the optical absorbance and transmittance of the MoSeTe monolayer. More importantly, due to the intrinsic asymmetric structure of Janus MoSeTe, we also consider the difference in optical responses on both side of the monolayer since light can interact with elements in unique ways, prompting different electron-photon interactions with different elements at the nanoscale. However, the obtained results help us to know that the two side surfaces show a similar sensitivity to a particular wavelength of light from infrared to visible colors to ultraviolet. Our findings not only unveil the basic optical properties of Janus MoSeTe, but also identify potential applications in designing ultra-thin reflectors and absorbers for optoelectronics in a certain frequency range for the MoSeTe monolayer.

\section{Computational methods}

The structural, electronic, and optical properties of Janus MoSeTe have been systematically studied by first-principles calculations based on density functional theory implemented in the Vienna Ab initio Simulation Package (VASP) code. ${ }^{29,30}$ The projector-augmented-wave (PAW) method is used to describe the interaction between valence electrons and ions. ${ }^{29}$ The exchange-correlation interactions are described by the generalized gradient approximation (GGA) with the Perdew-Burke-Ernzerhof $(\mathrm{PBE})^{31}$ to obtain the optimized $2 \mathrm{H}$ and $1 \mathrm{~T}$ configurations. It is wellknown that the standard DFT and semilocal approximation for the exchange correlation could not describe correctly the dispersive forces, and thus a nonlocal correction term should be added. To better describe this kind of dispersive force, Grimme's dispersion ${ }^{32}$ is used in our calculations. The valence electrons are described by a plane wave basis set with an energy cutoff of $520 \mathrm{eV}$. The Brillouin zone has been sampled with an $11 \times 11 \times 1 k$-mesh under the Monkhorst-Pack scheme ${ }^{33}$ to generate special $k$ points. After the optimized structures were obtained, the $17 \times 17 \times 1 k$ mesh has been used to determine the electronic density of states and optical properties. All of the structures are optimized until the HellmanFeynman forces are smaller than $0.01 \mathrm{eV} \AA^{-1}$. In addition, the Heyd-Scuseria-Ernzerhof (HSE) hybrid functional, ${ }^{34}$ including $25 \%$ of the Fock exchange and $75 \%$ of the Perdew, Burke, and Ernzerhof (PBE) exchange, ${ }^{35}$ is adopted to accurately describe the electronic and optical properties of the system. ${ }^{36}$ The spin-orbit coupling (SOC) effect is also considered throughout the band structure calculations. The optical properties of the Janus MoSeTe 
are also evaluated using $\mathrm{G}_{0} \mathrm{~W}_{0}$ (e-e correlation included and e-h correlation neglected) and the $\mathrm{G}_{0} \mathrm{~W}_{0}$ plus Bethe-Salpeter equation $\left(\mathrm{G}_{0} \mathrm{~W}_{0}+\mathrm{BSE}\right)$ approach (accounting for both e-e and e-h effects), respectively. Here, e-e and e-h represent the electron-electron and electron-hole correlation effects, respectively. For calculating the optical properties, we have used a $k$ mesh of $13 \times 13 \times 1$ to describe the Brillouin zone. $L_{z}=23.490 \AA$ and $L_{z}=23.623 \AA$ (the length of the unit cell along the $z$ direction) are applied for $2 \mathrm{H}$ and $1 \mathrm{~T}$ structures, respectively, to avoid the interactions between the periodic images of the surface. It is worth mentioning that spin-polarized and dipole corrections are also considered in all calculations. To better understand the structural stability, the phonon band structure and the phonon DOS are computed using a finite displacement approach with the help of the PHONOPY package. ${ }^{37}$ To have a profound understanding of charge transfer, the Bader charge approach ${ }^{38}$ is employed.

The optical absorption spectra can be determined from the frequency-dependent complex dielectric function $\varepsilon(\omega)=$ $\varepsilon_{1}(\omega)+\varepsilon_{2}(\omega)$ by DFT within the random phase approximation (RPA). ${ }^{39}$ Among them, the imaginary part of the dielectric function is determined by a summation over empty states from the Fermi golden rule: ${ }^{39}$

$$
\begin{aligned}
\varepsilon_{2}(\omega)= & \frac{4 \pi^{2} e^{2}}{\Omega} \lim _{q \rightarrow 0} \frac{1}{q^{2}} \sum_{c, \nu, \mathbf{k}} 2 \omega_{\mathbf{k}} \delta\left(\varepsilon_{c \mathbf{k}}-\varepsilon_{\nu \mathbf{k}}-\omega\right) \\
& \times\left\langle u_{c \mathbf{k}+e_{\alpha q}} \mid u_{\nu \mathbf{k}}\right\rangle \times\left\langle u_{c \mathbf{k}+e_{\alpha q}} \mid u_{\nu \mathbf{k}}\right\rangle^{*}
\end{aligned}
$$

where $c$ and $\nu$ are the conduction and valence band states, respectively. $u_{c \mathbf{k}}$ denotes the cell periodic part of the wavefunctions at the $k$ point. And the real part of the dielectric matrix is determined by the Kramers-Kronig transformation:

$$
\varepsilon_{1}(\omega)=1+\frac{2}{\pi} P \int_{0}^{\infty} \frac{\varepsilon_{2}\left(\omega^{\prime}\right)}{\omega^{\prime 2}-\omega^{2}+i \eta} \mathrm{d} \omega^{\prime}
$$

where $P$ is the principal value of the integral.

Then the main optical spectra, including the absorption coefficient $I(\omega)$, electron energy-loss spectrum $L(\omega)$, reflectivity $R(\omega)$, refractive index $\eta(\omega)$, and extinction coefficient $K(\omega)$, can be obtained from the dynamical dielectric response functions $\varepsilon(\omega)$. The specific expressions are given by:

$$
\begin{gathered}
I(\omega)=\sqrt{2} \omega\left[\sqrt{\varepsilon_{1}(\omega)^{2}+\varepsilon_{2}}(\omega)^{2}-\varepsilon_{1}(\omega)\right]^{1 / 2}, \\
L(\omega)=\omega_{2} /\left[\varepsilon_{1}(\omega)^{2}+\varepsilon_{2}(\omega)^{2}\right], \\
R(\omega)=\left|\frac{\sqrt{\varepsilon(\omega)}-1}{\sqrt{\varepsilon(\omega)}+1}\right|^{2}, \\
\eta(\omega)=(1 / \sqrt{2})\left[\sqrt{\varepsilon_{1}(\omega)^{2}+\varepsilon_{2}(\omega)^{2}}+\varepsilon_{1}(\omega)\right]^{1 / 2}
\end{gathered}
$$

and

$$
K(\omega)=(1 / \sqrt{2})\left[\sqrt{\varepsilon_{1}(\omega)^{2}+\varepsilon_{2}(\omega)^{2}}-\varepsilon_{1}(\omega)\right]^{1 / 2},
$$

respectively.

\section{Results and discussion}

\subsection{Geometry of the Janus MoSeTe monolayer}

The crystal structures of the Janus MoSeTe monolayer are shown in Fig. 1(a and b) with $2 \mathrm{H}$ and $1 \mathrm{~T}$ phases, respectively. Similar to parental $\mathrm{MoSe}_{2}, \mathrm{MoTe}_{2}$ and other transition-metal dichalcogenides (TMDs), the Janus MoSeTe monolayer has a graphene-like honeycomb lattice, ${ }^{40}$ where the metal Mo layer is sandwiched with two different kinds of chalcogen atoms, i.e., Se and Te atoms, with the sequence of Se-Mo-Te. Depending on the arrangement of chalcogen atoms, the traditional TMDs structure appears in two district symmetries: the $2 \mathrm{H}$ (trigonal prismatic $D_{3 \mathrm{~h}}$ ) phase and the $1 \mathrm{~T}$ (octahedral) phase. The $2 \mathrm{H}$ phase shows a hexagonal lattice with a threefold-symmetry. The $\mathrm{Se}-\mathrm{Mo} / \mathrm{Te}-\mathrm{Mo}$ atoms are in an eclipsing geometry from the top view, and hence the atomic stacking sequence is $\mathrm{AB}$, as shown in Fig. S1(a and b) for $\mathrm{MoSe}_{2}$ and $\mathrm{MoTe}_{2}$, respectively (ESI $\dagger$ ). However, the $1 \mathrm{~T}$ structure has an $\mathrm{ABC}$ stacking sequence in which the top Se/Te atoms occupy the hollow center of the $2 \mathrm{H}$ hexagonal lattice, as shown in Fig. S2 (ESI $\dagger$ ). Intriguingly, one of the unique properties of the MoSeTe monolayer is the Janus asymmetry where the structural imbalance in the MoSeTe monolayer breaks the traditional reflection symmetry along the out-of-plane direction, resulting in a spontaneous electric field within the monolayer. Also it is worth noting that the symmetry of Janus MoSeTe in the $2 \mathrm{H}$ phase is lower than those of the $\mathrm{MoSe}_{2}$ and $\mathrm{MoTe}_{2}$ monolayers though their structures are similar.

For the newly proposed 2D materials, we first calculated the phonon dispersions of the Janus MoSeTe monolayer to confirm their structural stability. Our calculations show that there are no imaginary frequencies in the phonon dispersions of the Janus MoSeTe monolayers in both $2 \mathrm{H}$ and $1 \mathrm{~T}$ phases, indicating that they are dynamically stable, as depicted in Fig. S3 (ESI $\dagger$ ). But the total energy calculations show that the $2 \mathrm{H}$ phase is lower than the $1 \mathrm{~T}$ phase by $0.113 \mathrm{eV}$ per unit, as shown in Fig. S4 (ESI $\dagger$ ). The calculated geometry and the lattice parameters of the optimized Janus MoSeTe are shown in Table 1 . The obtained lattice parameters of the $2 \mathrm{H}$ phase, i.e., $3.405 \AA$, is in agreement with the previous values of $3.394 \AA^{41}$ and $3.43 \AA^{42}$ Besides, the in-sheet lattice constant of $2 \mathrm{H}-\mathrm{MoSeTe}$ is only $0.06 \AA$ longer than that of the 1T-MoSeTe monolayer. In addition, we determine the
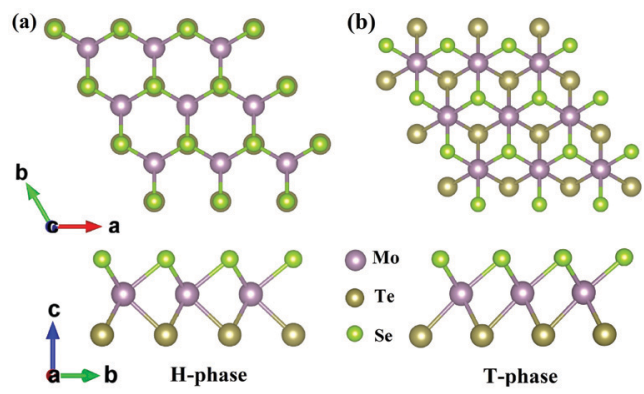

Fig. 1 Top (upper) and side (bottom) views of 2D Janus MoSeTe in (a) $2 \mathrm{H}$ and (b) $1 T$ phases, respectively. The purple, dark yellow, and green spheres represent Mo, Te and Se atoms, respectively. 
Table 1 Calculation of the lattice constants, Mo-Se and Mo-Te bond lengths, thickness, formation energies and band gap of Janus MoSeTe in $2 \mathrm{H}$ and $1 T$ phases, respectively

\begin{tabular}{llllllllll}
\hline & $a(\AA)$ & $d_{\mathrm{Mo}-\mathrm{Se}}(\AA)$ & $d_{\mathrm{Mo}-\mathrm{Te}}(\AA)$ & $h(\AA)$ & $E^{\mathrm{for}}(\mathrm{eV}$ per atom $)$ & $E_{\mathrm{g}}^{\mathrm{PBE}}(\mathrm{eV})$ & $E_{\mathrm{g}}^{\mathrm{PBE}+\mathrm{SOC}}(\mathrm{eV})$ & $E_{\mathrm{g}}^{\mathrm{HSE}}(\mathrm{eV})$ & $E_{\mathrm{g}}^{\mathrm{HSE}+\mathrm{SOC}}(\mathrm{eV})$ \\
\hline 2H-MoSeTe & 3.405 & 2.548 & 2.713 & 3.491 & -1.945 & 1.336 & 1.225 & 1.859 \\
1T-MoSeTe & 3.345 & 2.537 & 2.764 & 3.623 & -0.795 & 0.182 & 0.076 & 0.319 & 0.202
\end{tabular}

thicknesses of the $2 \mathrm{H}$ and $1 \mathrm{~T}$ MoSeTe monolayers to be $3.491 \AA$ and $3.623 \AA$, respectively. And the lengths of the Mo-Se (Mo-Te) bond are $2.548 \AA(2.713 \AA)$ and $2.537 \AA$ (2.764 $\AA$ ) with respect to $2 \mathrm{H}$ and $1 \mathrm{~T}$ phases, respectively. Accordingly, their formation energies $E^{\text {for }}$ are calculated by $E^{\text {for }}=E($ MoSeTe $)-N_{\mathrm{Mo}} E(\mathrm{Mo})-$ $N_{\mathrm{Se}} E(\mathrm{Se})-N_{\mathrm{Te}} E(\mathrm{Te})$, where $E(\mathrm{MoSeTe}), E(\mathrm{Mo}), E(\mathrm{Se})$, and $E(\mathrm{Te})$ are the energies of the MoSeTe monolayer, Mo, Se, and Te stable bulks, respectively. $N$ denotes the total number of specific atoms in a unit cell. Our calculations show that the $2 \mathrm{H}$-MoSeTe monolayer is energetically more favorable with $-1.945 \mathrm{eV}$ in energy lower than $-0.795 \mathrm{eV}$ for the 1T-MoSeTe monolayer, which is in agreement with the results of the total energy in Fig. S4 (ESI $\dagger$ ).

\subsection{Electronic structures of the Janus MoSeTe monolayer}

A precise evaluation of the electronic structure of Janus MoSeTe is indispensable in which the optical spectra are directly calculated from the interband transitions. Thus, the band structures of Janus MoSeTe along the highly symmetric $M-\Gamma-K-M$ path in the Brillouin zone are calculated by the HSE06, HSE + SOC, PBE and $\mathrm{PBE}+\mathrm{SOC}$ functionals and the results are shown in Fig. 2, Fig. S5 and S6 (ESI $\dagger$ ), respectively. Apparently, there are certain differences in the band structures of the $2 \mathrm{H}$ and $1 \mathrm{~T}$ phases. In the case of the 2H-MoSeTe monolayer in Fig. 2(a), we can find that it is a semiconductor with a direct band gap of $1.859 \mathrm{eV}$, which suggests that the $2 \mathrm{H}$ structure of Janus MoSeTe would be an excellent light harvesting 2D material. The HSE + SOC value is $1.726 \mathrm{eV}$, which is consistent with the previous value of $1.70 \mathrm{eV} .{ }^{42}$ The corresponding band gaps with PBE and PBE + SOC are $1.336 \mathrm{eV}$ and $1.225 \mathrm{eV}$ for the $2 \mathrm{H}$ structure, which are comparable with the previous results of $1.34 \mathrm{eV}$ and $1.22 \mathrm{eV}$ using GGA and GGA + SOC, respectively. ${ }^{41}$ It is worth noting that the gap of Janus MoSeTe in the $2 \mathrm{H}$ phase is in between the results of $\mathrm{MoS}_{2}$ and $\mathrm{MoTe}_{2} \cdot{ }^{43}$ The band gaps of the $1 \mathrm{~T}$ phase are $0.319 \mathrm{eV}$ and $0.202 \mathrm{eV}$ with respect to HSE and HSE + SOC functions. The corresponding PBE and PBE + SOC

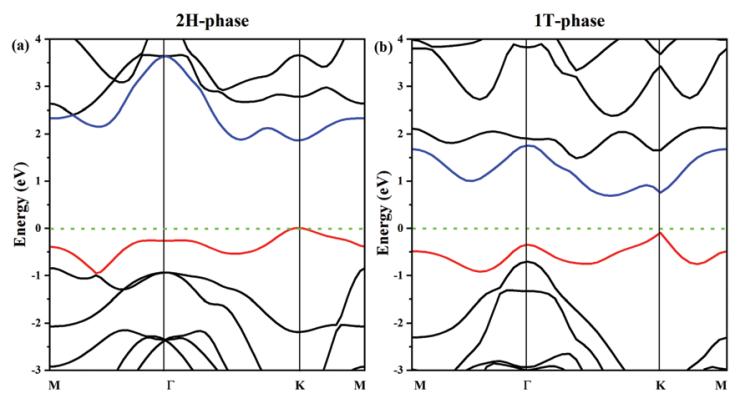

Fig. 2 Band structure of the Janus MoSeTe monolayer in (a) $2 \mathrm{H}$ and (b) $1 \mathrm{~T}$ phases, respectively, obtained using the hybrid functional HSE06. The dotted lines indicate the position of the Fermi level. results are $0.182 \mathrm{eV}$ and $0.076 \mathrm{eV}$, respectively. The details are listed in Table 1. We can see that SOC has little effects on the band gaps of the pristine MoSeTe structure. Remarkably, the top valence bands at the $K$ point are always higher than those at the $\Gamma$ point for the $2 \mathrm{H}$ phase, and the energy differences are respectively $274 \mathrm{meV}$ and $197 \mathrm{meV}$ as determined using HSE and PBE calculations, larger than the thermal energy at room temperature $\left(K_{\mathrm{T}} \sim 25.8 \mathrm{meV}\right)$, indicating that the electrons could be directly photoexcited from the valence band to the conduction band, which is feasible for the generation of photogenerated electrons and holes. Different from the case of the $2 \mathrm{H}$ structure, the maximum of the valence band and the minimum of the conduction band occur at the $K$ point and along the $\Gamma-K$ direction to the $1 \mathrm{~T}-\mathrm{MoSeTe}$ monolayer, respectively, resulting in an indirect-gap behavior. Besides, the band gap of the $1 \mathrm{~T}$ phase is smaller with a value of $0.3191 \mathrm{eV}$ at the HSE level, implying that 1T-MoSeTe is not an optically transparent material in which there will be amount of carriers created by absorption of light at room temperature.

In order to further elucidate the electronic properties of the Janus MoSeTe monolayer, the total density of states and the projected density of states for Mo $4 d$, Te $5 p$ and Se $4 p$ are calculated using the HSE functional, as depicted in Fig. 3. It is clearly shown that the valence bands are mainly contributed by Mo $4 \mathrm{~d}$ orbitals with a little Te $5 \mathrm{p}$ and Se $4 \mathrm{p}$ contribution in both $2 \mathrm{H}$ and $1 \mathrm{~T}$ phases. The Mo $4 \mathrm{~d}$ valence band covers from -6 to $0 \mathrm{eV}$ for the $2 \mathrm{H}$ phase and -7 to $0 \mathrm{eV}$ for the $1 \mathrm{~T}$ phase, respectively. Moreover, hybridization between Mo $4 \mathrm{~d}$ and Te $5 \mathrm{p}$ orbitals is larger than Mo $4 \mathrm{~d}$ and Se $4 \mathrm{p}$ orbitals under the
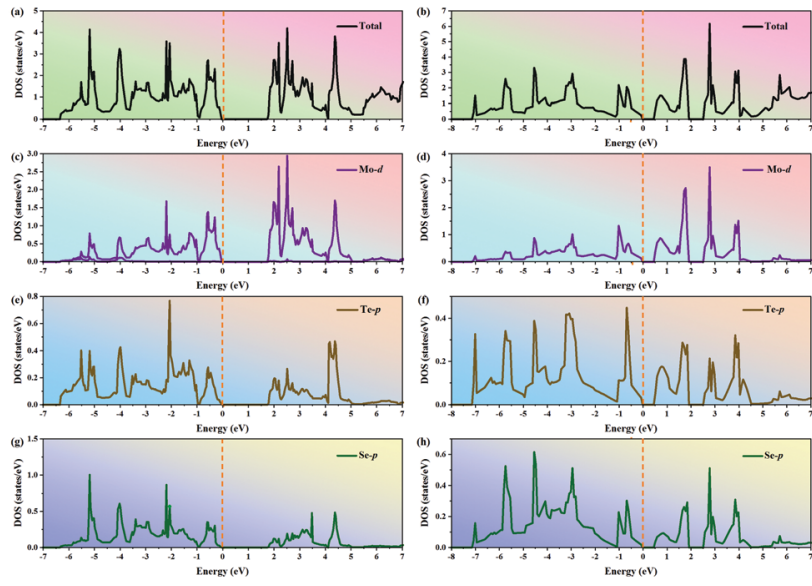

Fig. 3 Total DOS and projected density of states (PDOS) of the Janus MoSeTe monolayer in the $2 \mathrm{H}$ phase (left) and the $1 \mathrm{~T}$ phase (right), obtained using the hybrid functional HSE06. The dotted lines indicate the position of the Fermi level. 
Fermi level in both $2 \mathrm{H}$ and $1 \mathrm{~T}$ structures since the bandwidth of the valence bands for the Te atom is relatively larger than that of the Se atom. Regarding the unoccupied Mo 4d orbitals, an accurate description is also necessary for the interband transitions since electrons are generated from the occupied valence bands to the unoccupied conduction bands during optical excitations. For the $2 \mathrm{H}-\mathrm{MoSeTe}$ monolayer, the Mo $5 \mathrm{~d}$, Te $5 \mathrm{p}$ and Se $4 \mathrm{p}$ bands originate at about $1.84 \mathrm{eV}, 1.82 \mathrm{eV}$, and $1.78 \mathrm{eV}$, respectively. In contrast, the bands of the $1 \mathrm{~T}$ phase above the Fermi level originate at larger than $0.391 \mathrm{eV}$, as shown in Fig. 3(d, f and h) with respect to Mo, Te, and Se atoms, resulting in a smaller band gap. These results imply that the band gap is very sensitive to the arrangement of atoms along the in-plane direction and could be modulated by varying the interatomic separations and angles.

\section{Optical properties of the Janus MoSeTe monolayer}

In this section, we discuss the optical response of the Janus MoSeTe monolayer. The calculated dynamical dielectric functions using the HSE functional of the MoSeTe monolayer, including the imaginary part $\varepsilon_{2}(\omega)$ and real part $\varepsilon_{1}(\omega)$ of the dielectric function $\varepsilon(\omega)$ are displayed in Fig. 4 with respect to $2 \mathrm{H}$ and $1 \mathrm{~T}$ phases, whereas the optical spectra using the PBE functional is depicted in Fig. S7 (ESI $\dagger$ ). On the basis of structural anisotropy, the corresponding $\varepsilon(\omega)$ is split into three parts: $x x, y y$ and $z z$ directions with $\varepsilon^{x x}=\varepsilon^{y y} \neq \varepsilon^{z z}$, so we can see different behaviours when the incident photons are polarized the in-plane and out-of-plane of the $2 \mathrm{H}$-/1T-MoSeTe monolayer. The main difference between these spectra is that $\varepsilon(\omega)^{\|}$is more intense in the low range of $0-3.5 \mathrm{eV}$, whereas $\varepsilon(\omega)^{\perp}$ is more intense in the high range of energy $(4-11 \mathrm{eV})$ based on the observed blue shift for the threshold peaks of $\varepsilon(\omega)^{\perp}$.

The calculated static optical dielectric constants $\left(\varepsilon_{\propto}\right)$ in-plane are 4.939 and 19.359 for $2 \mathrm{H}$ and $1 \mathrm{~T}$ phases, respectively, which
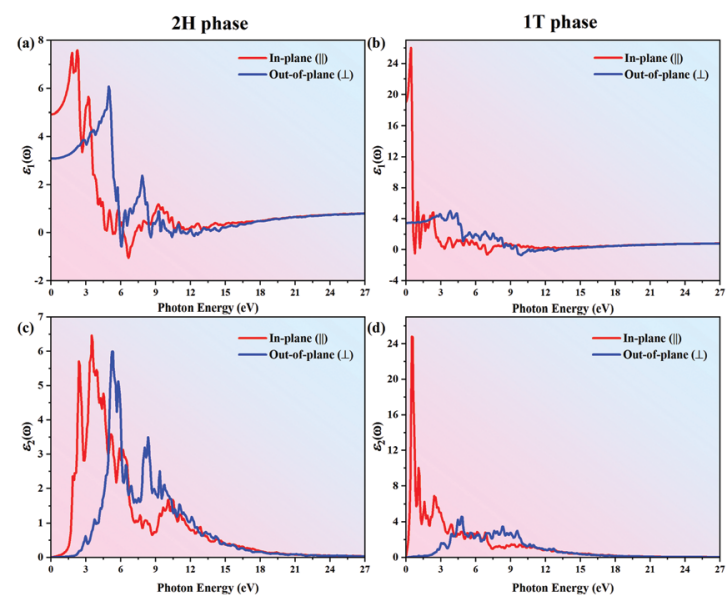

Fig. 4 The dynamical dielectric function $\varepsilon(\omega)=\varepsilon_{1}(\omega)+\varepsilon_{2}(\omega)$ as a function of the photon energy for the Janus MoSeTe monolayer in the $2 \mathrm{H}$ phase (left) and the 1T phase (right) obtained using the hybrid functional HSE06. turn to be 3.082 and 3.456 out-of-plane, indicating that 1T-MoSeTe has a relatively high polarizability. Besides, the negative values occur from $5.96 \mathrm{eV}$ to $7.36 \mathrm{eV}$ for the $2 \mathrm{H}$ phase, whereas they emerge at around $9.16 \mathrm{eV}$ and $10.00 \mathrm{eV}$ to the $1 \mathrm{~T}$ phase, as shown in Fig. 4(a and b) respectively, which unveils the metallic feature of the $2 \mathrm{H}$ and $1 \mathrm{~T}$ phases in the ultraviolet part of the electromagnetic spectrum. ${ }^{44}$

Regarding the imaginary part $\varepsilon_{2}(\omega)$, the first peak locates at $1.928 \mathrm{eV}(0.480 \mathrm{eV})$, similar to the band gap of $1.859 \mathrm{eV}$ $(0.391 \mathrm{eV})$ of the $2 \mathrm{H}(1 \mathrm{~T}) \mathrm{MoSeTe}$ construction, indicating that photoexcited electrons can directly transit from the VBM to the CBM. The second and third peaks locate at $2.337 \mathrm{eV}$ and $3.272 \mathrm{eV}$ for the $2 \mathrm{H}$ phase, respectively. The photon energy of the visible light ranges from 1.63 to $3.12 \mathrm{eV}$, suggesting that the $2 \mathrm{H}$-MoSeTe monolayer is a good light harvesting material. Besides, this visual light absorption is mainly contributed by the in-plane $\varepsilon_{2}^{x x}$ and $\varepsilon_{2}^{y y}$ components. It is noticed that there are several peaks for the out-of-plane $\varepsilon_{2}^{z z}$ of the $2 \mathrm{H}$ phase: at $2.92 \mathrm{eV}$, $3.74 \mathrm{eV}, 5.32 \mathrm{eV}, 8.36 \mathrm{eV}$, and $9.35 \mathrm{eV}$, showing that the $\varepsilon_{2}^{z z}$ cannot be neglected in the ultraviolet range. However, for the 1T phase, the second peak occurs at $0.960 \mathrm{eV}$. According to our calculated PDOS in Fig. 3(d), we suggest that this peak is assigned to the intra Mo $5 \mathrm{~d}$ transitions. Similarly, the $\varepsilon_{2}^{z z}$ component of the 1T phase makes an essential contribution between $2.72 \mathrm{eV}$ and $10.50 \mathrm{eV}$, which can be due to the electronic transitions from the $5 \mathrm{~d}$ states to the hybridized $5 \mathrm{~d} / 4 \mathrm{p}$ or $5 \mathrm{~d} / 5 \mathrm{p}$ orbitals.

On the other hand, the e-e correlation is considered to be the imaginary part $\varepsilon_{2}(\omega)$ of the dielectric function for Janus MoSeTe by using $\mathrm{G}_{0} \mathrm{~W}_{0}$ functions. Moreover, the $\mathrm{G}_{0} \mathrm{~W}_{0}$ plus Bethe-Salpeter equation (BSE) which introduces higher order interaction diagrams, i.e., e-e and e-h effects, is also considered to improve the electronic description systematically on top of $\mathrm{G}_{0} \mathrm{~W}_{0}{ }^{45,46}$ The obtained results are shown in Fig. S8 (ESI $\dagger$ ). It is clear that the optical anisotropy between $\varepsilon_{2}^{x x}$ (or $\varepsilon_{2}^{y y}$ ) and $\varepsilon_{2}^{z z}$ largely increases by the inclusion of local field effects, as shown in Fig. S8(a) vs. (c) or Fig. S8(b) vs. (d) (ESI $\dagger$ ). Additionally, the e-h interaction produces mainly a renormalization of the intensity of the optical peaks calculated using the $\mathrm{HSE}+\mathrm{G}_{0} \mathrm{~W}_{0}+\mathrm{BSE}$ function. More inspecting the imaginary part of the dielectric functions obtained at the HSE and $\mathrm{G}_{0} \mathrm{~W}_{0}$ levels for both phases, one can see that the inclusion of the e-e interaction leads to a blue shift. However, the inclusion of both e-e and e-h interactions yields a significant red shift, which is in agreement with the previous results. ${ }^{4-49}$ Another appealing peculiar result is that the first BSE optical peak is in much better agreement with the electronic gap than the other two calculated results, implying the weakly bound excitonic/free carrier nature of the optical excitation. Thus, the physical effect of the e-e and e-h interactions, reproduced by the HSE $+\mathrm{G}_{0} \mathrm{~W}_{0}+\mathrm{BSE}$ function, provides a more precise result. However, the total computation by $\mathrm{HSE}+\mathrm{G}_{0} \mathrm{~W}_{0}+\mathrm{BSE}$ is much more expensive than other methods. More importantly, it is worth mentioning that the global shape of the spectrum is preserved and calculated by these three methods for both $2 \mathrm{H}$ and $1 \mathrm{~T}$ phases of the MoSeTe monolayer. Considering the above factors, the optical properties of Janus MoSeTe could be well explained by independent-particle transitions. 


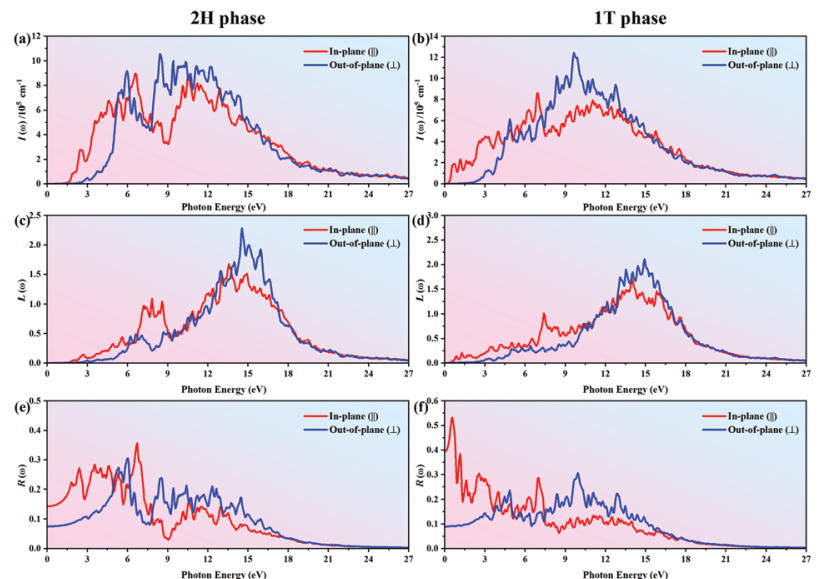

Fig. 5 Calculated absorption coefficient $/(\omega)$, the energy loss spectrum $L(\omega)$ and reflectivity $R(\omega)$ of the Janus MoSeTe monolayer in the $2 \mathrm{H}$ phase (left) and the 1T phase (right) obtained using the hybrid functional HSE06.

Fig. 5 shows the relationships of the adsorption coefficient $I(\omega)$, energy-loss $L(\omega)$ and the reflectivity $R(\omega)$ with photon energy for the Janus MoSeTe monolayer in the HSE functional obtained from the relation (eqn (3)-(5)) (see Fig. S9 for the comparison of PBE results, ESI $\dagger$ ). For the absorption coefficient $I(\omega)$ spectrum, which describes the decay of light traveling through the unit distance in an absorbing medium, there are many peaks within the studied energy range. The obtained $I(\omega)$ is zero when the photon energy is below $1.57 \mathrm{eV}$ and $0.35 \mathrm{eV}$ for $2 \mathrm{H}$ and $1 \mathrm{~T}$ phases, respectively. Correspondingly, it is located at $3.09 \mathrm{eV}$ and $1.22 \mathrm{eV}$ for $\mathrm{MoSe}_{2}$ and $\mathrm{MoTe}_{2}$ monolayers in the $2 \mathrm{H}$ phase, as shown in Fig. S13(a and b), respectively (ESI $\dagger$ ). From the $\mathrm{MoSe}_{2}$ to MoSeTe to $\mathrm{MoTe}_{2}$ monolayer in the $2 \mathrm{H}$ phase, we find that the red shift becomes stronger. This change is in good agreement with the trend of the band gap variance in which the minimum photon energy required for the electron interband transitions must be larger than the band gap. Thereafter, the adsorption coefficient gradually increases, implying that the absorption processes occur at the corresponding photon energies. Notably, the absorption coefficient could also describe the attenuation of intensity, i.e., the absorption spectra have a relatively low intensity with a small energy area since there are few electronic states that could play a role as the initial state in the absorption processes. Furthermore, the maximum peak of $I(\omega)$ could reach up to $1 \times 10^{6}$ at $8.53 \mathrm{eV}$ and $9.71 \mathrm{eV}$ for Janus MoSeTe in $2 \mathrm{H}$ and $1 \mathrm{~T}$ phases, respectively, implying high efficiency in the utilization of solar energy. Compared with the absorption coefficients $\left(<1 \times 10^{6}\right)$ of $\mathrm{MoS}_{2}{ }^{50}$ and MoSSe, ${ }^{51}$ we found that the obtained values are slightly larger than those of TMD materials. Besides, the obtained results are comparable to the values of $\mathrm{MoSe}_{2}$ and $\mathrm{MoTe}_{2}$, as shown in Fig. S13 (ESI $\dagger$ ). Additionally, the rest of the peaks occur at 2.69, 4.67, 9.35, $10.75 \mathrm{eV}$ for the $2 \mathrm{H}$ phase, and $0.59,3.18,6.88,12.76 \mathrm{eV}$ for the $1 \mathrm{~T}$ phase. These results show the same trend as that of the peaks of the imaginary part $\varepsilon_{2}(\omega)$ discussed above, confirming that the Janus MoSeTe monolayer mainly absorbs the visible and ultraviolet part of the electromagnetic spectrum.
Electrons in a medium can get excited by absorbing photons carrying energy. On the other hand, a fast traveling electron in a solid medium will release some energy, which may excite other electrons in the material. Besides, not only single particle excitations but also collective excitations occur, which can be identified by analyzing the energy-loss $L(\omega)$ spectrum related to the dielectric function using eqn (4). In the case of intraand interband transitions, $L(\omega)$ can demonstrate the plasmon excitations. The highest peak in the $L(\omega)$ spectrum indicates the characteristic associated with the plasma resonance and the corresponding highest frequency is the plasma frequency at which electrons could respond to. ${ }^{44,52}$ Thus, when electromagnetic waves have a lower frequency than the plasma frequency for the Janus MoSeTe monolayer, then the plasma oscillations will successfully screen the electric field since the electron has enough time to respond to the incident wave. As a result, the incident electromagnetic radiations are reflected from the surface of the Janus MoSeTe monolayer along with an increase in the reflection intensity. Hence, the peaks of $L(\omega)$ indicate the trailing edges in the reflection spectra. ${ }^{52}$ The maximum peak locates at $14.50 \mathrm{eV}$ and $14.97 \mathrm{eV}$ associated with $2 \mathrm{H}$ and $1 \mathrm{~T}$ phases, as shown in Fig. 5(c and d), respectively, indicating the plasmon resonance. Similarly, these peaks correspond to the abrupt reduction of the absorption coefficient $I(\omega)$ and reflectivity $R(\omega)$ in Fig. 5(e and f), respectively. Additionally, the origin of these peaks can also be explained according to the structure displayed in the real part of the dielectric function. As shown in Fig. 4(a and b), the real part $\varepsilon_{1}$ becomes zero at around $5.96 \mathrm{eV}(7.10 \mathrm{eV})$, arriving the minimum at about $6.72 \mathrm{eV}(9.94 \mathrm{eV})$, and again reaches zero at $14.97 \mathrm{eV}(13.43 \mathrm{eV})$ for the $2 \mathrm{H}(1 \mathrm{~T})$ phase. Regarding $\mathrm{MoSe}_{2}$ and $\mathrm{MoTe}_{2}$, from Fig. S13(c and d) (ESI $\dagger$ ), the highest peaks are located at $13.62 \mathrm{eV}$ and $15.40 \mathrm{eV}$, respectively, showing a blueshift from $\mathrm{MoSe}_{2}$ to MoSeTe to $\mathrm{MoTe}_{2}$. Notably, according to Schoenes's opinion, the energy where $\varepsilon_{1}$ crosses the zero line with a positive slope is the plasmon excitation energy. ${ }^{53}$ Moreover, when the incident electromagnetic radiation with a larger frequency passes through the plasma, the plasma oscillation frequency cannot follow the oscillations with less time to respond in the electric field, and therefore, no considerable change occurs in the incident wave, resulting in the disappearance of reflectivity $R(\omega)$. Subsequently, the electromagnetic wave could enter and pass through the 2D MoSeTe monolayer with the energy loss which is described by the absorption coefficient $I(\omega)$ and the electronic energy loss spectrum $L(\omega)$.

In the reflectivity $R(\omega)$ curves in Fig. 5(e and $\mathrm{f}$ ), there are many peaks up to $24 \mathrm{eV}$. The maximum reflectivity reaches $35 \%$ and $53 \%$ locating at $6.66 \mathrm{eV}$ and $0.478 \mathrm{eV}$ for the MoSeTe monolayer in $2 \mathrm{H}$ and $1 \mathrm{~T}$ phases, respectively. Particularly, the reflectivity of the $1 \mathrm{~T}$ phase is high in the IR region but decreases by about half to less than $30 \%$ in the visible and UV light regions, i.e., allowing UV and visible light to pass through the 1T-MoSeTe monolayer but reflecting the infrared radiation, resulting in limited heat levels with a minimum impact on the overall system performance. To the best of our knowledge, hot mirrors reflect IR radiation and transmit one or 

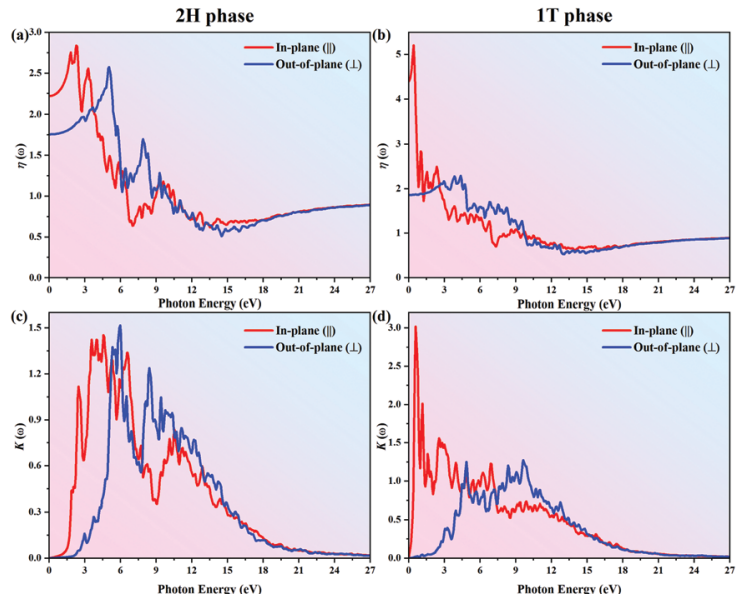

Fig. 6 Calculated refractive index $n(\omega)$ and extinction coefficient $K(\omega)$ for the Janus MoSeTe monolayer in the $2 \mathrm{H}$ phase (left) and the $1 \mathrm{~T}$ phase (right) obtained using the hybrid functional HSEO6.

more non-IR irradiation. Therefore, the 1T-MoSeTe monolayer is a good hot mirror material that can be used as a thermal reflective coating in the fields of energy/conservation and environmental protection. In contrast, reflectivity is around $30 \%$ for $\mathrm{MoSe}_{2}$, MoSeTe and $\mathrm{MoTe}_{2}$ in the $2 \mathrm{H}$ phase in the whole spectral region, leading to mediocre optical properties.

Fig. 6 shows the relationships of the refractive index $\eta(\omega)$ and the extinction coefficient $K(\omega)$ with photon energy of the proposed materials. The static refractive indices of the 2H-MoSeTe monolayer are 2.22 and 1.76 and reach peak values of 2.83 and 2.55 with respect to the in-plane and out-of-plane direction as shown in Fig. 6(a). Thereafter, the refractive indices decrease steadily and tend to be constant in the far UV region. Correspondingly, the static refractive indices of the $1 \mathrm{~T}$ phase are 4.484 and 1.861 for the in-plane and out-of-plane direction, respectively. But the difference of the peak values between them reaches up to 2.953, as clearly shown in Fig. 6(b). More obviously, $\eta^{\|}(\omega)$ decreases dramatically in the IR region, and when the photon energy exceeds $16 \mathrm{eV}$, it tends to be constant. The static refractive indices of $\mathrm{MoSe}_{2}$ and $\mathrm{MoTe}_{2}$, by contrast, are in the range of 1.94-2.47, but the peak values are 3.12 $\left(\mathrm{MoSe}_{2}\right), 3.25\left(\mathrm{MoTe}_{2}\right)$, respectively, which are larger than that of $2 \mathrm{H}-\mathrm{MoSeTe}$. For the extinction coefficient $K(\omega)$, it can be seen that the basic nature is the same for the in-plane and out-ofplane direction of the $2 \mathrm{H}$ phase with a blue-shift of maximally $2.70 \mathrm{eV}$ relative to the in-plane direction. A similar trend is also observed in $\mathrm{MoSe}_{2}$ and $\mathrm{MoTe}_{2}$ from Fig. S14 (ESI $\dagger$ ). However, in the case of 1T-MoSeTe, an additional peak appears in the spectrum at $0.504 \mathrm{eV}$ for the in-plane direction, and then the peak height drops sharply in the visible and ultraviolet region, which tends to be constant after $24 \mathrm{eV}$, as shown in Fig. 6(d). In broad terms, the large peak width of the extinction coefficient indicates that the photons could be absorbed quickly, since its depth path is the shortest. The penetration depth $(\delta=c / 2 \pi \omega K),{ }^{54}$ i.e., the skin depth, could correctly provide the penetration length at which the intensity of light or any electromagnetic radiation penetrating inside a material will fall to $1 / e$ or $37 \%$ of its original value at the surface. From $\mathrm{MoSe}_{2}$ to MoSeTe to $\mathrm{MoTe}_{2}$ in the $2 \mathrm{H}$ phase, the calculated maximum depths are around $29(22) \mathrm{nm}$, 25 (16) nm, and 24 (15) the in-plane (out-of-plane) direction, respectively. However, for the 1T-MoSeTe monolayer, it goes up to $117 \mathrm{~nm}(16 \mathrm{~nm})$. Since the thickness of these 2D monolayers is less than $0.4 \mathrm{~nm}$, the $\delta$ makes little sense.

The absorbance $A(\omega)$ of the Janus MoSeTe monolayer is calculated according to the Taylor expansion for a small thickness $\Delta z \rightarrow 0,55,56$

$$
A(\omega)=1-\mathrm{e}^{-\alpha \cdot \Delta z}
$$

Correspondingly, transparency $T(\omega)$ can be derived as,

$$
T(\omega)=1-R(\omega)-A(\omega) .
$$

The calculated results are shown in Fig. 7. One can see that there are many peaks at about 2.54, 4.65, 6.76, 8.46 and $12.36 \mathrm{eV}$ in the absorbance spectrum. The high absorbance $A(\omega)$ spectrum is about $2 \%$ in the visible range for both $2 \mathrm{H}$ and $1 \mathrm{~T}$ phases, which is comparable with the measured value of $2.8 \%$ for black phosphorus. ${ }^{57}$ Also one can see from Fig. S15 (ESI $\dagger$ ) that the integrated absorbance of the parental $\mathrm{MoSe}_{2}$ and $\mathrm{MoTe}_{2}$ is calculated to be a multiple of $3.55 \%$ and $4.44 \%$, which are larger than that of the Janus MoSeTe monolayer. In addition, the sharp absorbance peaks are observed around $8-9 \mathrm{eV}$ with $3.63 \%$ and $4.83 \%$ for $2 \mathrm{H}$ and $1 \mathrm{~T}$ MoSeTe, respectively. It is deduced that the MoSeTe monolayer can capture a significant fraction of sunlight in a subnanometer thickness. Additionally, it is observed that the overall absorbance magnitude is lower for the $2 \mathrm{H}$ phase than that of the $1 \mathrm{~T}$ phase. In contrast, the overall transmittance magnitude is higher for the Janus MoSeTe monolayer. In the ranges of $0-3.08 \mathrm{eV}, 8-10 \mathrm{eV}$ and $14-27 \mathrm{eV}$, the $2 \mathrm{H}$ MoSeTe monolayer is observed to transmit almost $90 \%$ of the incident light. Thus, we can predict that green, blue, violet and ultraviolet light are least absorbed, i.e., the most transmitted and absorbed in the Janus MoSeTe monolayer, indicating that the MoSeTe monolayer would be most easily seen with a green,
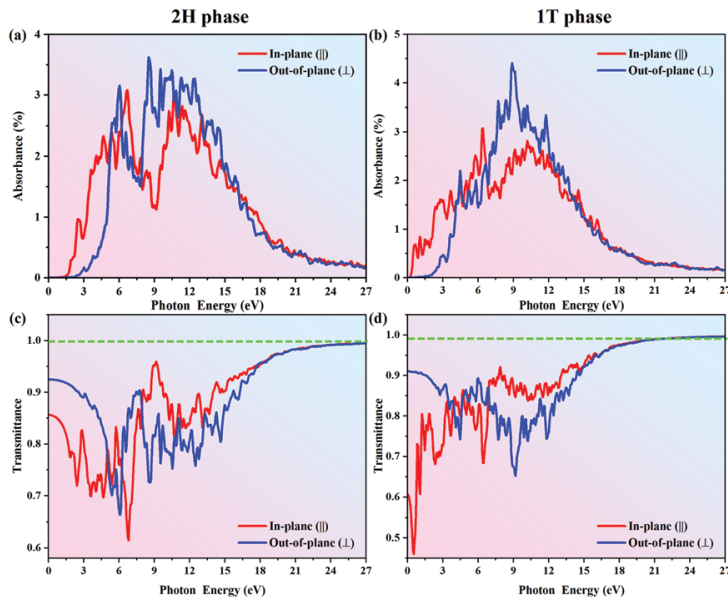

Fig. 7 Calculated absorbance and transmission spectrum for the Janus MoSeTe monolayer in the $2 \mathrm{H}$ phase (left) and the $1 \mathrm{~T}$ phase (right) obtained using the hybrid functional HSEO6. 
blue, and violet filter. Furthermore, it is observed that the MoSeTe monolayer generally transmits more red light than violet light. For comparison, the average optical transmittance in the region of $0.88-2.78 \mathrm{eV}$ is less than $80 \%$ for $\mathrm{MoSe}_{2}$, suggesting that it transmits more red, orange, yellow and green light, which is similar to $\mathrm{MoTe}_{2}$ in Fig. S15(d) (ESI $\dagger$ ).

We also studied the optical properties of Janus MoSeTe monolayer that sunlight goes through the 25 opposite orientation due to the structural anisotropy. Surprisingly, the shapes of the calculated optical spectra for different orientations exhibit almost the same main features. This might be because the slight difference in the optical thicknesses between $d_{\mathrm{Mo}-\mathrm{Se}}$ and $d_{\mathrm{Mo-Te}}$ for the MoSeTe monolayer results in a similar interaction between light with surface Se and Te atoms, which could be used in strengthening light reflection or transmission, and spectra splitting. In order to meet the higher requirements for optical characteristics, stacking systems should be used in which it can broaden the spectral range or bring about new functionalities, like polarizers. On the other hand, the greater intensities of light due to the multilayer coating could produce nonlinear effects, and accounting for these will certainly require further research. ${ }^{28}$

\section{Conclusions}

We have performed a comprehensive investigation of the electronic and optical properties of the Janus MoSeTe monolayer in $2 \mathrm{H}$ and $1 \mathrm{~T}$ phases, with a detailed comparison of the parental $\mathrm{MoSe}_{2}$ and $\mathrm{MoTe}_{2}$ monolayer, through first-principles calculations based on DFT. Firstly, our calculations show that Janus MoSeTe in $2 \mathrm{H}$ and $1 \mathrm{~T}$ structures exhibit dynamical stability and high experimental feasibility. Besides, we demonstrate that the $2 \mathrm{H}$ structure shows a direct bandgap of $1.859 \mathrm{eV}$ and an indirect gap of $0.391 \mathrm{eV}$ for the $1 \mathrm{~T}$ structure, which triggers plasmons absorbing light at different frequencies. Besides, the SOC has little effects on the band gaps. Analytically, we obtain microscopic insights into the origins of the maximum optical response which shows a significant red shift from the $\mathrm{MoSe}_{2}$ to MoSeTe to MoTe 2 monolayer. However, the blue-shift exists from the in-plane to out-of-plane direction. Compared with the HSE results, it is found that the inclusion of the $\mathrm{e}-\mathrm{e}$ interaction led to a blue shift of $\varepsilon_{2}(\omega)$. However, the inclusion of both $\mathrm{e}-\mathrm{e}$ and $\mathrm{e}-\mathrm{h}$ interactions results in a significant red shift. Phenomenologically, 1T-MoSeTe interacts with light strongly from the infrared to visible to ultraviolet regions due to its small band gap nature. More importantly, the maximum reflectivity of the $1 \mathrm{~T}$ phase reaches up to $53 \%$ at $0.478 \mathrm{eV}$, enabling it to be a good hot mirror material. In addition, the absorption coefficient values could reach up to $1 \times 10^{6} \mathrm{~cm}^{-1}$ in both phases, implying the high efficiency in the utilization of solar energy of the MoSeTe monolayer. Additionally, the average optical absorbance of the Janus MoSeTe monolayer in the visible light region is calculated to be $2 \%$ and the corresponding transmittance is around $80 \%$. Furthermore, the optical response orientated to the opposite surface is also considered in our work due to the intrinsic asymmetric structure of Janus MoSeTe. Surprisingly, our results show a similar sensitivity to a particular wavelength of light in different optical ranges. Our findings not only predict the great potential application of Janus MoSeTe in ultra-thin reflectors and absorbers for optoelectronic devices, but also may provide guidelines to the discovery of new optical science and the realization of various light emission, detection, modulation, and manipulation functions in specific frequency ranges.

\section{Conflicts of interest}

There are no conflicts to declare.

\section{Acknowledgements}

We sincerely acknowledge the financial support from the National Natural Science Foundation of China (Grant No. 11705152, 11625415, and 41272050), the Joint Fund of the National Natural Science Foundation of China and the China Academy of Engineering Physics (Grant No. U1530258), the Science Challenge Project (Grant No. TZ2016001), the National Magnetic Confinement Fusion Energy Research Project of China (Grant No. 2015B108002), and the Longshan academic talent research supporting program of Southwest University of Science and Technology (Grant No. 18LZX513 and 18LZXT04). And the authors gratefully acknowledge financial support from the China Scholarship Council. DS and RA thank Carl Tryggers Stiftelse for Vetenskaplig Forskning (CTS) for financial support. SNIC and HPC2N are acknowledged for providing the computing facilities.

\section{References}

1 S. Das Sarma, S. Adam, E. H. Hwang and E. Rossi, Rev. Mod. Phys., 2011, 83, 407-470.

2 K. S. Novoselov, A. K. Geim, S. V. Morozov, D. Jiang, Y. Zhang, S. V. Dubonos, I. V. Grigorieva and A. A. Firsov, Science, 2004, 306, 666-669.

3 R. R. Nair, P. Blake, A. N. Grigorenko, K. S. Novoselov, T. J. Booth, T. Stauber, N. M. Peres and A. K. Geim, Science, 2008, 320, 1308.

4 G. Eda and S. A. Maier, ACS Nano, 2013, 7, 5660-5665.

5 A. Splendiani, L. Sun, Y. Zhang, T. Li, J. Kim, C. Y. Chim, G. Galli and F. Wang, Nano Lett., 2010, 10, 1271-1275.

6 N. Alem, R. Erni, C. Kisielowski, M. D. Rossell, W. Gannett and A. Zettl, Phys. Rev. B: Condens. Matter Mater. Phys., 2009, 80, 155425.

7 C. R. Dean, A. F. Young, I. Meric, C. Lee, L. Wang, S. Sorgenfrei, K. Watanabe, T. Taniguchi, P. Kim, K. L. Shepard and J. Hone, Nat. Nanotechnol., 2010, 5, 722-726.

8 H. Liu, A. T. Neal, Z. Zhu, Z. Luo, X. Xu, D. Tománek and P. D. Ye, ACS Nano, 2014, 8, 4033-4041.

9 M. Xu, T. Liang, M. Shi and H. Chen, Chem. Rev., 2013, 113, 3766-3798. 
10 M. Chhowalla, H. S. Shin, G. Eda, L. J. Li, K. P. Loh and H. Zhang, Nat. Chem., 2013, 5, 263.

11 J. N. Coleman, M. Lotya, A. O. Neill, S. D. Bergin, P. J. King, U. Khan, K. Young, A. Gaucher, S. De, R. J. Smith, I. V. Shvets, S. K. Arora, G. Stanton, H. Y. Kim, K. Lee, G. T. Kim, G. S. Duesberg, T. Hallam, J. J. Boland, J. J. Wang, J. F. Donegan, J. C. Grunlan, G. Moriary, A. Shmeliov, R. J. Nicholls, J. M. Perkins, E. M. Grieveson, D. W. McComb, K. Theuwissen, P. D. Nellist and V. Nicolosi, Science, 2011, 331, 568-571.

12 B. Anasori, M. R. Lukatskaya and Y. Gogotsi, Nat. Rev. Mater., 2017, 2, 16098.

13 L. Tao, E. Cinquanta, D. Chiappe, C. Grazianetti, M. Fanciulli, M. Dubey, A. Molle and D. Akinwande, Nat. Nanotechnol., 2015, 10, 227.

14 M. Dávila, L. Xian, S. Cahangirov, A. Rubio and G. Le Lay, New J. Phys., 2014, 16, 095002.

15 S. Balendhran, S. Walia, H. Nili, S. Sriram and M. Bhaskaran, Small, 2015, 11, 640-652.

16 A. J. Mannix, X. F. Zhou, B. Kiraly, J. D. Wood, D. Alducin, B. D. Myers, X. Liu, B. L. Fisher, U. Santiago, J. R. Guest, M. J. Yacaman, A. Ponce, A. R. Oganov, M. C. Hersam and N. P. Guisinger, Science, 2015, 350, 1513-1516.

17 D. Singh, S. K. Gupta, Y. Sonvane and I. Lukačević, RSC Adv., 2016, 6, 8006-8014.

18 X. H. Liu, C. Z. Guan, D. Wang and L. J. Wan, Adv. Mater., 2014, 26, 6912-6920.

19 L. Dou, A. B. Wong, Y. Yu, M. Lai, N. Kornienko, S. W. Eaton, A. Fu, C. G. Bischak, J. Ma, T. Ding, N. S. Ginsberg, L. W. Wang, A. P. Alivisatos and P. Yang, Science, 2015, 349, 1518-1521.

20 R. Ma and T. Sasaki, Adv. Mater., 2010, 22, 5082-5104.

21 R. Ma and T. Sasaki, Acc. Chem. Res., 2014, 48, 136-143.

22 A. Y. Lu, H. Zhu, J. Xiao, C. P. Chuu, Y. Han, M. H. Chiu, C. C. Cheng, C. W. Yang, K. H. Wei, Y. Yang, Y. Wang, D. Sokaras, D. Nordlund, P. Yang, D. A. Muller, M. Y. Chou, X. Zhang and L. J. Li, Nat. Nanotechnol., 2017, 12, 744.

23 J. Zhang, S. Jia, I. Kholmanov, L. Dong, D. Er, W. Chen, H. Guo, Z. Jin, V. B. Shenoy and L. Shi, et al., ACS Nano, 2017, 11, 8192-8198.

24 Q. F. Yao, J. Cai, W. Y. Tong, S. J. Gong, J. Q. Wang, X. Wan, C. G. Duan and J. Chu, Phys. Rev. B, 2017, 95, 165401.

25 L. Dong, J. Lou and V. B. Shenoy, ACS Nano, 2017, 11, 8242-8248.

26 F. Li, W. Wei, H. Wang, B. Huang, Y. Dai and T. Jacob, J. Phys. Chem. Lett., 2019, 10, 559-565.

27 X. Yuan, M. Yang, L. Wang and Y. Li, Phys. Chem. Chem. Phys., 2017, 19, 13846-13854.

28 S. Gupta, S. N. Shirodkar, A. Kutana and B. I. Yakobson, ACS Nano, 2018, 12, 10880-10889.

29 B. Hammer, L. B. Hansen and J. K. Nørskov, Phys. Rev. B: Condens. Matter Mater. Phys., 1999, 59, 7413.

30 G. Kresse and J. Furthmüller, Phys. Rev. B: Condens. Matter Mater. Phys., 1996, 54, 11169.

31 J. Perdew, K. Burke and M. Ernzerhof, Phys. Rev. Lett., 1996, 77, 3865-3868.
32 S. Grimme, J. Antony, S. Ehrlich and H. Krieg, J. Chem. Phys., 2010, 132, 154104.

33 H. Monkshort and J. Pack, Phys. Rev. B: Solid State, 1976, 13, 5188-5192.

34 J. Heyd, G. E. Scuseria and M. Ernzerhof, J. Chem. Phys., 2003, 118, 8207-8215.

35 J. P. Perdew, A. Ruzsinszky, G. I. Csonka, O. A. Vydrov, G. E. Scuseria, L. A. Constantin, X. Zhou and K. Burke, Phys. Rev. Lett., 2008, 100, 136406.

36 N. Bickers, D. Scalapino and S. White, Phys. Rev. Lett., 1989, 62, 961.

37 A. Togo, F. Oba and I. Tanaka, Phys. Rev. B: Condens. Matter Mater. Phys., 2008, 78, 134106.

38 E. Sanville, S. D. Kenny, R. Smith and G. Henkelman, J. Comput. Chem., 2007, 28, 899-908.

39 M. Gajdoš, K. Hummer, G. Kresse, J. Furthmüller and F. Bechstedt, Phys. Rev. B: Condens. Matter Mater. Phys., 2006, 73, 045112.

40 C. Ataca, M. Topsakal, E. Aktürk and S. Ciraci, J. Phys. Chem. C, 2012, 116, 8983-8999.

41 S. D. Guo and J. Dong, Semicond. Sci. Technol., 2018, 33, 085003.

42 Y. Ji, M. Yang, H. Lin, T. Hou, L. Wang, Y. Li and S. T. Lee, J. Mater. Chem. C, 2018, 122, 3123-3129.

43 S. D. Guo and J. L. Wang, Semicond. Sci. Technol., 2016, 31, 095011.

44 D. Singh, S. K. Gupta, Y. Sonvane and I. Lukačević, J. Mater. Chem. C, 2016, 4, 6386.

45 M. Rohlfing and S. G. Louie, Phys. Rev. Lett., 1998, 81, 2312.

46 G. Kresse and J. Furthmüller, Comput. Mater. Sci., 1996, 6, 15-50.

47 Z. Guan, S. Ni and S. Hu, J. Phys. Chem. C, 2018, 122, 6209-6216.

48 H. Jin, T. Wang, Z. R. Gong, C. Long and Y. Dai, Nanoscale, 2018, 10, 19310-19315.

49 F. Karlicky and M. Otyepka, J. Chem. Theory Comput., 2013, 9, 4155-4164.

50 N. N. Hieu, V. V. Ilyasov, T. V. Vu, N. A. Poklonski, H. V. Phuc, L. T. Phuong, B. D. Hoi and C. V. Nguyen, Superlattices Microstruct., 2018, 115, 10-18.

51 X. Ma, X. Wu and Y. Wang, J. Mater. Chem. A, 2018, 6, 2295-2301.

52 F. Wooten, Optical properties of solids, Academic Press, 1973, vol. 41, pp. 939-940.

53 J. Schoenes, Phys. Rep., 1980, 63, 301-336.

54 D. B. Tanner, Optical effects in solids, Cambridge University Press, 2019.

55 M. Bernardi, M. Palummo and J. C. Grossman, Nano Lett., 2013, 13, 3664-3670.

56 L. Yang, J. Deslippe, C. H. Park, M. L. Cohen and S. G. Louie, Phys. Rev. Lett., 2009, 103, 186802.

57 A. Castellanos Gomez, L. Vicarelli, E. Prada, J. O. Island, K. Narasimha-Acharya, S. I. Blanter, D. J. Groenendijk, M. Buscema, G. A. Steele, J. Alvarez, H. W. Zandbergen, J. J. Palacios and H. S. J. V. D. Zant, 2D Mater., 2014, 1, 025001. 